(1)

CrossMark

\title{
Noninvasive versus invasive pressure- flow relationship of the pulmonary circulation: bias and error
}

\author{
To the Editor:
}

Testing the pulmonary circulation in dynamic conditions has been proposed in recent years as a means to provide additional insights into patients' symptoms and disease severity, complementing diagnostic evaluations at rest [1-6]. As pulmonary vascular haemodynamics can be described as a function of flow, resistance and left atrial pressure, it has been suggested that pressure-flow relationship of the pulmonary circulation can discriminate normal from abnormal behaviours, the latter consistent with cardiac, pulmonary or pulmonary vascular disease [4].

Echocardiography can provide an accurate noninvasive estimate of pulmonary haemodynamics, but may be of limited precision [7-9]. Although this tool is particularly suitable for population studies (being noninvasive and having no systematic bias), it has insufficient precision for clinical decision making, which does not obviate the need for invasive confirmation. Indeed, while accuracy and precision of echocardiography have been repeatedly proven for measurements at rest [7-9], much less evidence is available regarding exercise data [10-13], with only one report focusing on the pressure-flow relationship [11]. Thus, although the theoretical advantage of using a noninvasive approach by echocardiography cannot be denied, there is paucity of information on whether it might provide adequate estimates of pressure-flow relationships during exercise.

The aim of the present study was to compare echocardiographic estimates and invasive measurements of the pressure-flow relationship for the pulmonary circulation. In particular, and in keeping with analyses of data obtained in resting conditions [7-9], we hypothesised that exercise stress echocardiography would be rather accurate compared to invasive measurements, but might suffer from limited precision.

We included patients undergoing a clinically indicated right heart catheterisation (RHC), which was followed immediately by invasive haemodynamic evaluation as well as by echocardiographic evaluation during exercise. The analysis was approved by the local ethics committees and patients gave consent for the use of their data for research purposes. Patients were recruited in two centres (Erasme Hospital, Brussels, Belgium and Istituto Auxologico Italiano, Milan, Italy) at which the protocol described was followed.

We excluded patients who did not have a good-quality tricuspid regurgitant jet signal at Doppler echocardiography, thus precluding pulmonary artery pressure (PAP) estimation.

Zero reference was placed at midthoracic level [1]. Upon completion of resting haemodynamic measurements, the patient's feet were placed on the pedals and, after a 2-min rest, subjects were asked to exercise in the supine position. Workload was increased in a stepwise manner every 2-3 min by 5-30 W, according to the subject's exercise capacity, in order to achieve at least three steps of exercise, and measurements were taken during the last minute of each step $[1,3,5]$. Invasive haemodynamic recordings were analysed offline by two operators blinded to patients' clinical characteristics and echocardiographic data. Pressure values were averaged over several heart beats (at least eight) and over several respiratory cycles [1].

@ERSpublications

Exercise echocardiography can provide imprecise but rather accurate and sensitive estimates of pressure-flow relationships of the pulmonary circulation, as compared with RHC under exercise http://bit.ly/2YQIPZ1

Cite this article as: Caravita S, Yerly P, Baratto C, et al. Noninvasive versus invasive pressure-flow relationship of the pulmonary circulation: bias and error. Eur Respir J 2019; 54: 1900881 [https://doi.org/ 10.1183/13993003.00881-2019]. 
Methodology for stress echocardiographic assessment of the pulmonary circulation during exercise has been described previously [2, 14]. In brief, subjects exercised in a supine (Erasme) or semi-supine (Auxologico) position. Workload was increased in a stepwise manner every 2-3 min (Erasme) or using a ramp protocol (Auxologico), with measurements taken every 2-3 min. All echocardiographic measurements were performed offline by a single operator, blinded to patients' clinical characteristics and RHC data.

Mean PAP was plotted against cardiac output and the linear regression slope relating these two variables throughout the exercise test (P/Q slope) was calculated both for RHC and for echocardiographic data [1, $4,14]$. In addition, the ratio between mean PAP and cardiac output at peak exercise (total pulmonary vascular resistance (TPR) at peak) was computed $[1,3]$.

Accuracy and precision of echocardiographic estimates of pressure-flow relationships of the pulmonary circulation, compared with invasive measurements, were evaluated using Bland-Altman analysis. The bias, standard deviation of the difference, and 95\% limits of agreement were reported. All data analyses were performed using R Core Team software (2016; Vienna, Austria).

After excluding 20 subjects ( $\mathrm{n}=18$ with suboptimal tricuspid regurgitation signal and $\mathrm{n}=2$ with severe tricuspid regurgitation precluding PAP estimation by echocardiography; $80 \%$ without pulmonary
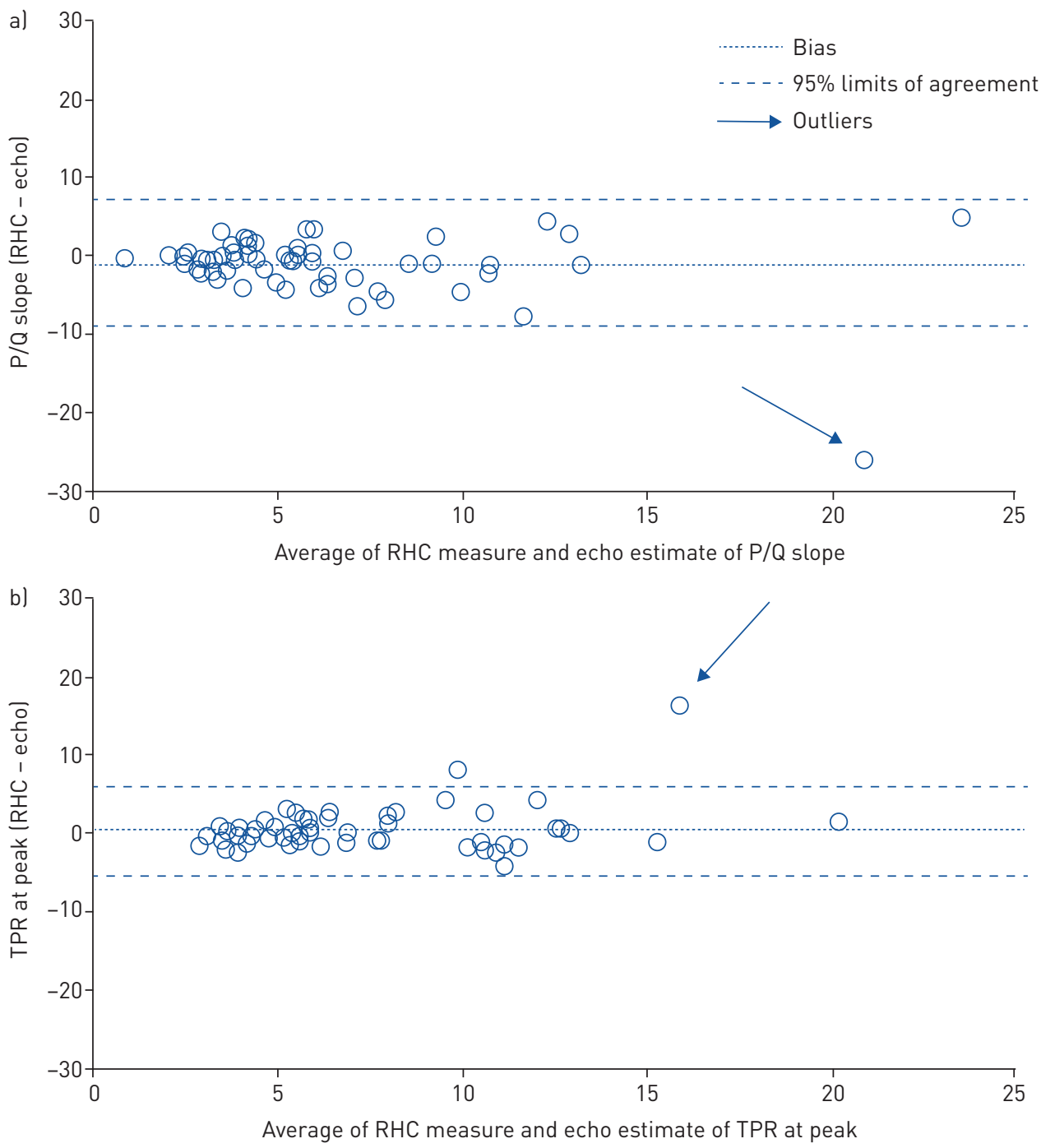

FIGURE 1 Bland-Altman plots representing the bias and limits of agreement of echocardiographic estimates as compared with invasive measurements of al the regression slope between mean pulmonary artery pressure and cardiac output (P/Q slope) and b) total pulmonary vascular resistance (TPR) at peak exercise. $\mathrm{RHC}$ : right heart catheterisation. 
hypertension at rest), our final population consisted of 60 subjects ( $73 \%$ female). All of the included subjects had at least three pairs of pressure and flow invasive measurements and echocardiographic estimates, including peak values.

$63 \%$ of patients had pulmonary hypertension at rest, which was precapillary in $61 \%$ of cases. Nevertheless, when considering in addition patients without pulmonary hypertension at rest, more than half of the patients (55\%) suffered from left heart failure, with a preserved left ventricular ejection fraction in $70 \%$ of cases. Only $7 \%$ of patients were on permanent atrial fibrillation. $27 \%$ of patients had a chronic obstructive pulmonary disease (Global Initiative for Chronic Obstructive Lung Disease (GOLD) stage 1-2 in 63\% of cases).

Echocardiographic estimates of $\mathrm{P} / \mathrm{Q}$ slope and TPR at peak were significantly correlated with invasive measurements $\left(\mathrm{R}^{2}=0.38\right.$ and 0.56 , respectively; $\left.\mathrm{p}<0.001\right)$. The mean differences between echocardiographic estimates and invasive measurements of TPR at peak and P/Q slope were -0.4 and $1.2 \mathrm{mmHg} \cdot \mathrm{L}^{-1} \cdot \mathrm{min}^{-1}$, respectively (figure 1). However, the $95 \%$ limits of agreement for echocardiographic estimates of TPR at peak and $\mathrm{P} / \mathrm{Q}$ slope were quite large $\left(5.7\right.$ and $8.2 \mathrm{mmHg} \cdot \mathrm{L}^{-1} \cdot \mathrm{min}^{-1}$, respectively). These results were consistent when separately analysing the subgroup of patients $(n=30)$ which underwent both exercise echocardiography and exercise RHC in the supine position, which included $90 \%$ of patients with pulmonary hypertension at rest (mean difference between echocardiographic estimates and invasive measurements of TPR at peak and P/Q slope -0.9 and $1.4 \mathrm{mmHg} \cdot \mathrm{L}^{-1} \cdot \mathrm{min}^{-1}$, respectively; $95 \%$ limits of agreement for echocardiographic estimates of TPR at peak and P/Q slope 7.6 and $11.3 \mathrm{mmHg} \cdot \mathrm{L}^{-1} \cdot \mathrm{min}^{-1}$, respectively).

Echocardiographic estimates of $\mathrm{P} / \mathrm{Q}$ slope and of TPR at peak were more imprecise and inaccurate in patients with obesity or with lung disease $(n=26)$. The mean differences between echocardiographic estimates and invasive measurements of TPR at peak and P/Q slope were -0.8 and $2.2 \mathrm{mmHg} \cdot \mathrm{L}^{-1} \cdot \mathrm{min}^{-1}$, respectively, in patients with obesity or lung disease, and 0.3 and $-0.1 \mathrm{mmHg} \cdot \mathrm{L}^{-1} \cdot \mathrm{min}^{-1}$ in patients without these comorbidities. The 95\% limits of agreement for echocardiographic estimates of TPR at peak and $\mathrm{P} / \mathrm{Q}$ slope were 10.9 and $7.7 \mathrm{mmHg} \cdot \mathrm{L}^{-1} \cdot \mathrm{min}^{-1}$, respectively, in patients with obesity or lung disease, and 4.8 and $3.6 \mathrm{mmHg} \cdot \mathrm{L}^{-1} \cdot \mathrm{min}^{-1}$, respectively, in patients without these comorbidities.

Furthermore, after subdividing the population by the median of the difference between invasive measurement and echocardiographic estimate, P/Q slope was more imprecise in patients with obesity $(\mathrm{p}<0.001)$, in those with higher mean PAP values $(\mathrm{p}<0.01)$, lower cardiac index $(\mathrm{p}<0.05)$ and higher pulmonary vascular resistance $(p<0.01)$. TPR was more imprecise in patients with higher mean PAP $(\mathrm{p}=0.06)$ and higher pulmonary vascular resistance $(\mathrm{p}<0.01)$.

When considering the subgroup of patients $(n=22)$ without pulmonary hypertension at rest, the mean differences between echocardiographic estimates and invasive measurements of TPR at peak and P/Q slope were $0.8 \mathrm{mmHg} \cdot \mathrm{L}^{-1} \cdot \mathrm{min}^{-1}$ for both variables, while the $95 \%$ limits of agreement for echocardiographic estimates of TPR at peak and P/Q slope were 1.9 and $2.9 \mathrm{mmHg} \cdot \mathrm{L}^{-1} \cdot \mathrm{min}^{-1}$, respectively.

Our study provides one of the first evaluations of the accuracy and precision of echocardiographic estimates of pressure-flow relationships of the pulmonary circulation during exercise, as compared to RHC. In keeping with previous analyses $[11,12]$, noninvasive pressure-flow relationships seem to have a reasonably good agreement with invasive measurements. However, some aspects deserve attention. First, our data show that echocardiography provides an imprecise estimate of pressure-flow relationships of the pulmonary circulation, not only at rest, but also during exercise. Many possible explanations have been already proposed to account for the imprecision of Doppler echocardiography at rest, which is mirrored by the dispersion of individual points in Bland-Altman plots [7-9, 11]. These may include suboptimal quality of Doppler signals, imprecise estimation of right atrial pressure, as well as the reliance on few Doppler signal recordings (in inspiration or in expiration) according to the acoustic window available rather than based on an average over several respiratory cycles. All these factors are likely to hold true for exercise evaluations, and might be particularly relevant for patients predisposed to have huge intrathoracic pressure swings, such as obese patients and patients with lung disease, as well as for patients with higher PAP values. Indeed, the 95\% limits of agreement for echocardiographic estimates were quite large, especially when considering that the cut-off value to separate normal from abnormal responses has been suggested to be $\sim 3 \mathrm{mmHg} \cdot \mathrm{L}^{-1} \cdot \mathrm{min}^{-1}$ for both variables $[2,5,6]$. Even if echocardiography seemed to perform slightly better in the subgroup of patients without pulmonary hypertension at rest (95\% limits of agreement for echocardiographic estimates \pm 2.9 for P/Q slope and \pm 1.9 for TPR at peak), its imprecision might render its interpretation questionable in these most delicate cases [15]. Furthermore, PAP estimation was not possible in one-fourth of consecutively evaluated patients, including a large number of patients with normal pulmonary haemodynamics at rest. Overall, our data suggest a bias towards echocardiographic overestimation of pressure-flow relationships of the pulmonary circulation. The bias 
was minimal for TPR at peak, but possibly relevant for P/Q slope, which incorporates multiple points and might thus be more prone to accumulation of errors.

Some limitations of our work should be acknowledged. First, this study was conducted on a relatively small number of highly selected patients, i.e. patients with a clinical indication to undergo RHC after a thorough clinical evaluation. However, proposing RHC for research purposes only in this context would be deemed unethical [1]. Half of the patients underwent exercise echocardiography in the semi-supine position. However, body position seemed not to affect our results. In this regard, it has been shown previously that neither maximal exercise capacity [16, 17] nor P/Q slope relationship [17] might be relevantly affected by body position. Finally, both exercise echocardiography and exercise RHC are time-consuming techniques, which were blindly and rigorously analysed, eventually providing results that are coherent with what should have been expected, based on previous suggestions.

In conclusion, our results suggest that, when adequate tricuspid regurgitant jet signal is present, exercise echocardiography can provide imprecise but rather accurate and sensitive estimates of pressure-flow relationships of the pulmonary circulation, as compared with RHC under exercise. Therefore, such an intrinsic imprecision of echocardiography should probably limit its use in the assessment of the pulmonary circulation in clinical practice.

Sergio Caravita $\oplus^{1,2,3,5}$, Patrick Yerly ${ }^{4,5}$, Claudia Baratto ${ }^{1,2}$, Céline Dewachter ${ }^{3}$, Andrea Faini $\oplus^{1}$, Asma Rimouche ${ }^{3}$, Giovanna Branzi ${ }^{1}$, Giovanni Battista Perego $\odot^{1}$, Antoine Bondue ${ }^{3}$, Gianfranco Parati ${ }^{1,2}$ and Jean-Luc Vachiéry ${ }^{3}$ ${ }^{1}$ Istituto Auxologico Italiano, IRCCS, Ospedale San Luca, Milan, Italy. ${ }^{2}$ Dept of Medicine and Surgery, University of Milano-Bicocca, Milan, Italy. ${ }^{3}$ Dept of Cardiology, Cliniques Universitaires de Bruxelles, Hôpital Académique Erasme, Brussels, Belgium. ${ }^{4}$ Dept of Cardiology, Centre Hospitalier Universitaire Vaudois, Lausanne, Switzerland. ${ }^{5}$ Both authors contributed equally.

Correspondence: Jean-Luc Vachiéry, Dept of Cardiology, CUB Hôpital Erasme, 808 Route de Lennik, 1070 Brussels, Belgium. E-mail: jeanluc.vachiery@erasme.ulb.ac.be

Received: 3 May 2019 | Accepted after revision: 12 Aug 2019

Conflict of interest: None declared.

Support statement: S. Caravita is the recipient of an ERS PAH Short-Term Research Training Fellowship (STRTF 2014-5264) supported by an unrestricted grant by GSK, and of the international grant "Cesare Bartorelli" in 2014 funded by the Italian Society of Hypertension. A. Bondue received support from the Belgian Fonds de la Recherche Scientifique (FRS-FNRS - grant J.0011.19), from the Erasme Foundation (ULB) and from the Belgian Cardiac Surgery Foundation. J-L. Vachiery is the holder of the Actelion Research Chair on Pulmonary Hypertension in his department. Funding information for this article has been deposited with the Crossref Funder Registry.

\section{References}

1 Kovacs G, Herve P, Barbera JA, et al. An official European Respiratory Society statement: pulmonary haemodynamics during exercise. Eur Respir J 2017; 50: 1700578.

2 Rudski LG, Gargani L, Armstrong WF, et al. Stressing the cardiopulmonary vascular system: the role of echocardiography. J Am Soc Echocardiogr 2018; 31: 527-550.

3 Herve P, Lau EM, Sitbon O, et al. Criteria for diagnosis of exercise pulmonary hypertension. Eur Respir J 2015; 46 : 728-737.

4 Naeije R, Vanderpool R, Dhakal BP, et al. Exercise-induced pulmonary hypertension: physiological basis and methodological concerns. Am J Respir Crit Care Med 2013; 187: 576-583.

5 Borlaug BA, Nishimura RA, Sorajja P, et al. Exercise hemodynamics enhance diagnosis of early heart failure with preserved ejection fraction. Circ Heart Fail 2010; 3: 588-595.

6 Eisman AS, Shah RV, Dhakal BP, et al. Pulmonary capillary wedge pressure patterns during exercise predict exercise capacity and incident heart failure. Circ Heart Fail 2018; 11: e004750.

7 D'Alto M, Romeo E, Argiento P, et al. Accuracy and precision of echocardiography versus right heart catheterization for the assessment of pulmonary hypertension. Int J Cardiol 2013; 168: 4058-4062.

8 Rich JD, Shah SJ, Swamy RS, et al. Inaccuracy of Doppler echocardiographic estimates of pulmonary artery pressures in patients with pulmonary hypertension: implications for clinical practice. Chest 2011; 139: 988-993.

9 Fisher MR, Forfia PR, Chamera E, et al. Accuracy of Doppler echocardiography in the hemodynamic assessment of pulmonary hypertension. Am J Respir Crit Care Med 2009; 179: 615-621.

10 Obokata M, Kane GC, Reddy YN, et al. Role of diastolic stress testing in the evaluation for heart failure with preserved ejection fraction: a simultaneous invasive-echocardiographic study. Circulation 2017; 135: 825-838.

11 Claessen G, La Gerche A, Voigt JU, et al. Accuracy of echocardiography to evaluate pulmonary vascular and RV function during exercise. JACC Cardiovasc Imaging 2016; 9: 532-543.

12 van Riel AC, Opotowsky AR, Santos M, et al. Accuracy of echocardiography to estimate pulmonary artery pressures with exercise: a simultaneous invasive-noninvasive comparison. Circ Cardiovasc Imaging 2017; 10: e005711.

13 Kovacs G, Maier R, Aberer E, et al. Assessment of pulmonary arterial pressure during exercise in collagen vascular disease: echocardiography $v s$ right-sided heart catheterization. Chest 2010; 138: 270-278.

14 Argiento $\mathrm{P}$, Chesler N, Mulè M, et al. Exercise stress echocardiography for the study of the pulmonary circulation. Eur Respir J 2010; 35: 1273-1278. 
15 Vachiéry JL, Tedford RJ, Rosenkranz S, et al. Pulmonary hypertension due to left heart disease. Eur Respir J 2019; 53: 1801897.

16 Reddy YNV, Olson TP, Obokata M, et al. Hemodynamic correlates and diagnostic role of cardiopulmonary exercise testing in heart failure with preserved ejection fraction. JACC Heart Fail 2018; 6: 665-675.

17 Forton K, Motoji Y, Deboeck G, et al. Effects of body position on exercise capacity and pulmonary vascular pressure-flow relationships. J Appl Physiol 2016; 121: 1145-1150.

Copyright @ERS 2019 